\title{
The Track Sheet
}

Dr. Drake's habit of recording the precise details of a patient's clinical history, angiographic findings, treatment, and results along with a pencil tracing of the angiogram was well known to all of his residents and fellows. He called them his "track sheets" and they formed the basis for his critical analysis of operative methods and results over the years. Many of the vascular neurosurgeons he trained have continued this tradition. When asked several years ago, he said that it came mostly from Sir Hugh Cairns in Oxford, who probably observed it during his time with Cushing in Boston. The accumulated track sheets of over 1700 posterior circulation aneurysms comprise a permanent record of an experience unlikely to be repeated in vascular neurosurgery, and that will continue to be a valuable resource for many years to come.

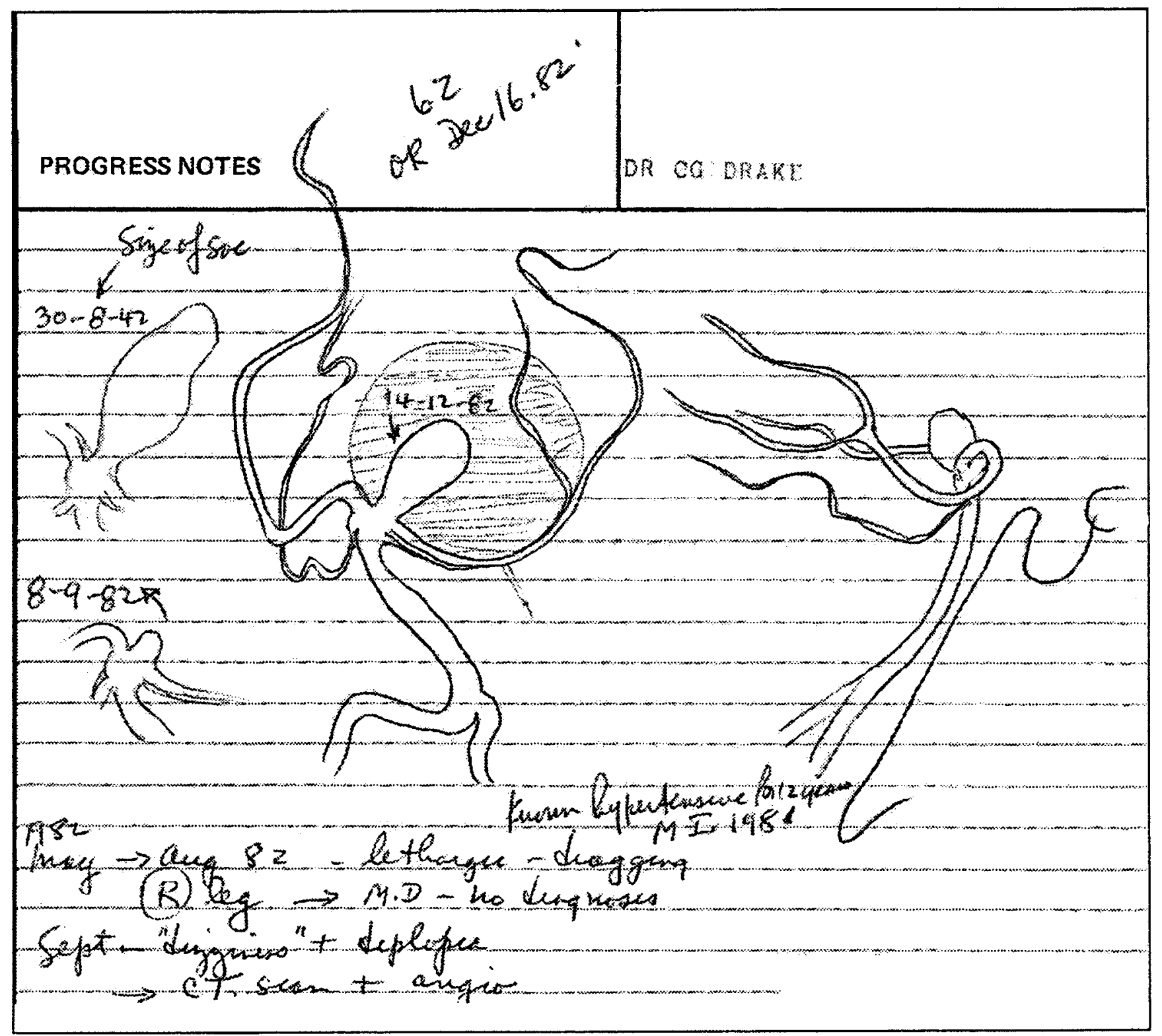

